\title{
Finite Volume/Mixed Finite Element Analysis of Pollutant Transport and Bioremediation in Heterogeneous Saturated Aquifers
}

\author{
CLAUDIO GALLO ${ }^{1,2}$ AND GIANMARCO MANZINI ${ }^{3, *}$ \\ ${ }^{1}$ CRS 4, Zona Industriale Macchiareddu, Uta, Cagliari - Italy \\ 2 Dept. of Civil Engineering and Geosciences, TUDelft, Delft, - The Netherlands \\ ${ }^{3}$ IAN - CNR, via Ferrata 1, 27100 Pavia, - Italy
}

\begin{abstract}
SUMMARY
The adoption of a suitable pumping-injecting well network and the human enhancement of the activity of soil bacteria, whose metabolism contributes to degrade and transform many pollutants in non-toxic substances, may be crucial in the process of remediation of contaminated soils. Organic contaminant transport in a subsurface aquifer and its biological degradation kinetics is numerically addressed by using a four contaminant species model. A numerical approach is proposed, that is based on a cell-center finite volume method for the system of advection-dispersion equations of contaminants with a mixed-hybrid finite element method for the solution of a single-phase Darcy's equation. The effectiveness of the method and its accuracy in retaining the main physical properties of the continuous mathematical model is illustrated by simulating the time evolution of contaminant concentrations in a set of realistic scenarios. Copyright (c) 2000 John Wiley \& Sons, Ltd.
\end{abstract}

KEY WORDS: bioremediation, contaminant transport, mixed finite elements, finite volumes

\section{INTRODUCTION}

Soil contamination has recently become a problem of major social concern, because a wide range of pollutant agents of different chemical nature and toxicity may be present in subsurface aquifers. Pollution sources are either accidental events, like spills and leaks, or common human activities, like disposal of urban sewage, industrial wastes, and the use of pesticides and fertilizers in agriculture, see References [24, 25, 22, 20, 9, 8]. The contaminants in a subsurface aquifer are subject to complex physical and chemical processes, such as dispersion, advection by groundwater flow, chemical reactions and biological degradation due to soil microorganisms.

The groundwater flow is described by a single phase Darcy's equation, while the subsurface transport of different chemical species are modeled by a set of coupled advection-dispersionreaction equations [3].

${ }^{*}$ Correspondence to: Gianmarco Manzini, IAN - CNR, via Ferrata 1, 27100 Pavia, Italy 
The biological degradation depends on the microorganism population whose metabolism is affected by the availability in soils of substrates like organic carbon, electron acceptors oxygen and nitrogen - and nutrients $[17,19]$.

The organic carbon needed to sustain bacterial life is naturally present in soil, while nutrients - such as phosphates, nitrates, ammonia - may be provided also by human input, for instance fertilizers. The bacterial population is normally stable because it dynamically tends to an equilibrium state in which its growth rate is balanced by its decay rate. When the concentration of nutrients augments due to an external supply, the bacterial population increases of several orders of magnitude and tends to a new equilibrium state. The remarkable fact is that the bacterial metabolic processes may effectively reduce hazardous organic pollutants to harmless byproducts, such as $\mathrm{CO}_{2}$ and $\mathrm{H}_{2} \mathrm{O}$ [1]. In this context, a remediation strategy can be devised which relies on the enhancement of the biodegradation activity, see Reference [23] for a literature review on this topic. The biodegradation kinetics models proposed in literature are usually classified in three distinct classes, respectively termed free-bacteria, microcolony-based and biofilm models $[16,11]$.

The simplest models belong to the first class [18]. They basically assume that bacteria exist as individual particles within the aqueous phase or adsorbed by soil grains. No assumption is made on the microscopic configuration and distribution of bacteria in soil pores, and on the way the organisms are grouped together on the solid pore surface. These latter facts are considered irrelevant for the macroscopic description of bacterial population growth and decay.

In the second class of models, bacteria do not exist as individual particles but in small discrete colonies, or microcolonies, attached to the soil grain surfaces. Growth and decay of the biomass contained in microcolonies are formulated either by taking that the bacterial colony dimension can grow by consumption of organic substrate and electron acceptors or by assuming the colony dimension constant and varying their concentration, i.e., the number of colonies per unit volume [21].

The main feature of the models in the third class is that the solid particles constituting the aquifer material are covered by a biofilm within which consumption of the substrates and electron-acceptors takes place $[26,16,11]$. The key processes are the mass exchange between bulk flow and the biofilm and the internal degradation of organic substrates.

A more detailed discussion of the similarities and differences between these models is beyond the scope of the present work. We refer the interested reader to the discussion in Reference [2] see also the bibliography therein - where it is shown that under a set of simplifying assumptions the three approaches reduce to an essentially equivalent description of the biodegradation process. This, however, is true only for very simple cases.

Our approach relies on the four-species model documented in References [21, 27] in the context of the more general microcolony-based concept. The main feature of this model lies in its capability of describing how the metabolism of subsurface microbes can be enhanced by concurrent metabolization of oxygen, nitrogen and nutrients. From the computational viewpoint, it is a compromise between the simpler free-bacteria model which tends to overestimate the degradation extent, and the more accurate but also more complex and expensive bio-film model.

The numerical approximation of the complete mathematical model is a research issue, and many different aspects make the numerical simulation of a bioremediation process challenging. We mention in the following list the topics we feel the most significant and that we consider in the present work. 
- Treatment of highly heterogeneous soil: the value of the permeability can differ for four orders of magnitude or more in two adjacent mesh cells.

- Advection-dominated transport: the model species concentrations in the groundwater bulk-flow can feature strong gradient regions when sharp concentrations fronts move throughout the computational domain.

- Non-linear coupling effects, even if the soil is a saturated one. The kinetics of the bacterial population depends on the contaminants which diffuse within the microcolonies from the groundwater bulk flow. It also exerts its influence on the bulk-flow contaminant concentrations via a set of reactive source terms in the transport equations.

These issues have already been investigated by the authors in these previous works.

In [4] we presented some preliminary results on the discretization of the flow and transport equations by using mixed finite elements and finite volumes.

The coupling of the contaminant transport equations with a bacterial population equation and its numerical discretization was investigated in [12]. In this work the model species are passively advected by a constant velocity and pressure fields. In order to solve the Darcy's equation we adopted a high-order accurate mixed finite element scheme $\left(\mathcal{B D}_{\mathcal{D} \mathcal{N}_{1}}\right)$. Despite is accuracy, this approach is not appropriate to simulate non-linear phenomena requiring a frequent update of the velocity and pressure fields because of the high computational cost.

A better approach from this viewpoint is based on the mixed-hybrid scheme proposed in [13]. In this work we validated the method on the standard quarter-of-five-spots problem, focusing the attention on the treatment of the soil heterogeneity.

Finally, the work presented in this paper copes with the bioremediation of a field-size aquifer that has been contaminated by an accidental leak out. Different bioremediation techniques and human intervention strategies are numerically investigated to predict the clean-up time for an almost complete removal of pollutants.

According with our previous experience we propose the following numerical approach. The steady groundwater bulk flow is approximated by using the lowest-order mixed-hybrid finite element method. This approach yields an approximation to the steady velocity field that is more accurate than the one provided by straightforward differentiation of the conforming finite element approximation of the pressure field. In particular, we emphasize that the mixed-hybrid finite element method ensure local - i.e. cell-wise - mass conservation, while the conforming finite element approximation lacks local mass conservation. The contaminant transport equations are approximated in space by an unstructured triangle-based finite volume method and advanced in time by a semi-implicit two-stage Runge-Kutta scheme. A TVD stability condition is imposed by a multidimensional limiting procedure. The resulting scheme is formally second-order accurate, conservative, and capable of capturing strong solution gradient fronts moving at the correct physical propagation speeds.

The species interactions are taken into account in the full-species model by solving iteratively their non-linear interaction coupling.

The outline of the paper follows. In Section 2, we review the mathematical model describing single-phase bulk flow, contaminant transport and bacterial kinetics. The discretization method is summarized in Section 3. We address here the finite volume discretization of the contaminant transport equations as well as the mixed-hybrid finite element approximation of the Darcy's phase pressure and velocity fields. In Section 4 we present the results of a set of numerical investigations that assess the performance of possible remediation strategies. We consider also 
different networks of extraction/injection wells, whose running mode has been selected on the basis of the plume location and the soil remediation status. The conclusions follow in Section 5 .

\section{THE MATHEMATICAL MODEL}

athematical_model

\subsection{Transport Equations}

Transport phenomena are mathematically described by a system of $N_{D S}$ coupled advectiondispersion-reaction equations, where $N_{D S}$ is the number of dissolved species. In divergence form they can be written as follows

$$
R_{i} \frac{\partial C_{i}}{\partial t}+\operatorname{div}\left(\mathbf{u} C_{i}-\mathbf{D}_{i}(\mathbf{u}) \nabla C_{i}\right)=B_{i}, \quad i=1, \ldots, N_{D S}
$$

eq: transport

The variables $C_{i}$ in equations (1) represent the bulk flow concentration of each transported species; the terms $R_{i}$ are the retardation factors, which take into account chemical adsorption processes, the terms $\mathbf{D}_{i}(\mathbf{u})$ are the velocity-dependent dispersion tensors. The r.h.s. source terms $B_{i}$ describe the coupling between the species concentrations transported in the bulk flow and the ones within microcolonies. Also equations (1) are supplemented by appropriate boundary conditions, such as inlet, outlet and no-flow, and initial solution states to specify the application problems.

\subsection{The Darcy's Equation}

Groundwater bulk flow in an heterogeneous saturated soil is mathematically formulated by the Darcy's equation [10]

$$
\left\{\begin{aligned}
\mathbf{u} & =-\mathbf{K} \nabla p, & & \text { in } \Omega \\
\operatorname{div} \mathbf{u} & =f, & & \text { in } \Omega .
\end{aligned}\right.
$$

The pressure field is indicated by $p$ and the groundwater velocity field by $\mathbf{u}, \mathbf{K}(\mathbf{x})$ is the transmissivity tensor, and $f(\mathbf{x})$ a source/sink term. Equations (2) are completed by a set of suitable boundary conditions of Neumann/Dirichlet-type, modelizing inlet/outlet and no-flow boundary configurations.

\subsection{The Bioremediation Model}

Microcolony-based models assume that bacteria reside and act within microcolonies, described as a set of patches attached to soil grains [21]. From the bulk phase, chemical species can reach microcolonies via diffusive mass exchange. Depending on the mass-transfer coefficient, concentrations within microcolonies govern the degradation rate kinetics and can be significantly different from those in the bulk phase.

In this class of models, the term $B_{i}$ of equation (1) is expressed in terms of a diffusive mass exchange from the bulk to the microcolony phase,

$$
B_{i}=N_{c} \kappa_{i} A_{c} \frac{\left(C_{i}-c_{i}\right)}{\delta}, \quad i=1, \ldots, N_{D S}
$$


where $\kappa_{i}$ is the mass-exchange coefficient between bulk flow and microcolonies, $A_{c}$ is the contact area of one microcolony for the mass diffusion process, $\delta$ is the thickness of the boundary layer between bulk flow and microcolonies, $c_{i}$ is the concentration of the component $i$ in the microcolonies and $N_{c}$ is the number of microcolonies per unit volume.

The assumption that the biodegradation process works essentially at a steady-state regime yields the following form for the r.h.s. terms $B_{i}$ in (3):

$$
\kappa_{i} A_{c} \frac{\left(C_{i}-c_{i}\right)}{\delta}=\mu_{0, i} m_{c} \sum_{k=1}^{N_{E A}} Y_{i, k}\left[\prod_{j=1}^{N_{D S}^{i}} \frac{c_{j}}{K_{j, k}+c_{j}}\right] I_{b}^{k-1}+Q_{i} I_{b}^{i-1}, \quad i=1, \ldots, N_{D S}
$$

where $\mu_{0, i}$ are the maximum rate coefficients, $m_{c}$ is the mass of a microcolony, $Y_{i, k}$ are the yield coefficients which account for the stoichiometry and efficiency of degradation, $K_{j, k}$ are the half saturation constants, and $I_{b}^{k-1}$ are the inhibition functions [21]. In equation (4) the symbol $N_{E A}$ denotes the number of electron acceptors and $N_{D S}^{i}$ the number of dissolved species involved in the degradation of the $i$-th species. The term $Q_{i}$ is non zero only when the component $i$ is an electron acceptor - for instance oxygen or nitrate. In this case, it takes the form

$$
Q_{i}=\alpha_{i} \frac{c_{i}}{K_{i}+c_{i}}, \quad i=1, \ldots, N_{E A},
$$

where $\alpha_{i}$ is the electron acceptor coefficient for the maintainance energy of bacteria, and $K_{i}$ is the electron acceptor saturation constant.

This degradation equation states that the total amount of a compound entering a microcolony in a given interval of time is equal to the amount of species that is degraded in the same interval. The rate of degradation and consequently the concentration within microcolonies, is roughly proportional to the concentration outside the colonies. The terms $Q_{i}$ introduce into the model the consumption of oxygen due to bacterial decomposition [21, 27] as a first order decay term.

Bacterial kinetics is modeled by the following time-dependent differential equation that describes the microcolony population dynamics,

$$
\frac{1}{N_{c}} \frac{\partial\left(\phi N_{c}\right)}{\partial t}=\sum_{i=1}^{N_{D H}}\left[\mu_{0, i} \sum_{k=1}^{N_{E A}} Y_{i, k}\left(\prod_{j=1}^{N_{D S}^{i}} \frac{c_{j}}{K_{j, k}+c_{j}}\right)\right]-k_{d}^{c}
$$

where $k_{d}^{c}$ is the population decay constant, $N_{D H}$ is the number of dissolved hydrocarbons organic substrates - and $\phi$ is the porosity of the medium $[21,27]$.

\section{THE NUMERICAL MODEL}

\subsection{The Finite Volume Discretization of the Transport Equations}

The numerical discretization in the framework of the finite volume scheme is defined on the same mesh $\mathcal{T}_{h}(\Omega)$ used for the mixed-hybrid scheme of the previuous section. The index $h$ is the maximum diameter of the $N_{T}$ triangles forming the mesh, i.e. $h=\max _{T \in \mathcal{T}_{h}(\Omega)} h_{T}$, where $h_{T}$ is the lenght of the longest edge of the triangle $T$. As usual, these triangulations are assumed regular and conformal for $h \rightarrow 0$ in the sense specified in Reference [7], pag. 132. 
Equations (1) are reformulated in a cell-wise integral form by integrating on each triangular cell $T$ and then applying the Gauss divergence theorem to transform the spatial divergence term into a balance of edge integral fluxes. Let us introduce for every $T \in \mathcal{T}_{h}(\Omega)$ the vector $\mathbf{U}_{T}$, whose elements are the cell-averaged concentrations of the transported species,

$$
\left.\mathbf{U}_{T}\right|_{i}=\frac{1}{|T|} \int_{T} C_{i} d T .
$$

The semi-discrete finite volume approximation is

$$
\begin{aligned}
|T| \mathbf{R} \frac{d \mathbf{U}_{T}}{d t}+ & \sum_{e \in \sigma(T)} \mathbf{G}_{e}\left(\mathbf{u}_{e}, \widetilde{\mathbf{U}}_{T}, \widetilde{\mathbf{U}}_{T_{e}}, \mathbf{n}_{e}\right)+\sum_{e \in \sigma(T)} \mathbf{H}_{e}\left(\mathbf{u}_{e}, \widetilde{\mathbf{U}}_{T}, \widetilde{\mathbf{U}}_{T_{e}}, \mathbf{n}_{e}\right) \\
& +\sum_{e \in \sigma^{\prime}(T)} \mathbf{F}_{e}^{(b c)}=\sum_{q} \omega_{T, q} \mathbf{S}_{T}\left(\widetilde{\mathbf{U}}_{T}\left(\mathbf{x}_{T, q}\right)\right), \quad \text { for every } T \in \mathcal{T}_{h}(\Omega),
\end{aligned}
$$

where the diagonal matrix $\mathbf{R}=\operatorname{diag}\left(R_{1}, \ldots, R_{N_{D S}}\right)$ collects the retardation factors, and for every cell $T$,

- $|T|$ is the measure of its area, and $\partial T$ its boundary;

- $\sigma(T)$ is the subset of its internal edges; these latters are the edges that $T$ shares with an adjacent mesh cell indicated by $T_{e}$, so that for every $e \in \sigma(T)$ there exists a cell $T_{e} \in \mathcal{T}_{h}(\Omega)$ such that $e=\partial T \cap \partial T_{e} ;$

- $\sigma^{\prime}(T)$ is the subset of the edges of $T$ located at the boundary of the computational domain; that is, for every $e \in \sigma^{\prime}(T)$ we have $e=\partial T \cap \partial \Omega$.

The cell interface flux integral is evaluated by using suitable advective and dispersive numerical fluxes across the edge $e$, that are

$$
\begin{aligned}
\left.\mathbf{G}_{e}\left(\mathbf{u}_{e}, \widetilde{\mathbf{U}}_{T}, \widetilde{\mathbf{U}}_{T_{e}}, \mathbf{n}_{e}\right)\right|_{i} & \left.\approx \int_{e} \mathbf{n} \cdot \mathbf{u} \mathbf{U}\right|_{i} d l \\
\mathbf{H}_{e}\left(\mathbf{u}_{e}, \widetilde{\mathbf{U}}_{T}, \widetilde{\mathbf{U}}_{T_{e}}, \mathbf{n}_{e}\right) & \left.\left.\approx \int_{e} \mathbf{n} \cdot \mathbf{D}\right|_{i}(\mathbf{u}) \nabla \mathbf{U}\right|_{i} d l
\end{aligned}
$$

and the numerical flux function $\mathbf{F}_{e}^{(b c)}$ at boundary edges.

The numerical flux vector functions $\mathbf{G}_{e}$ and $\mathbf{H}_{e}$ introduced in (9) depend on $\mathbf{u}_{e}$, which is the value of the velocity field $\mathbf{u}$ at the midpoint of the edge $e$ shared by the triangles $T$ and $T_{e}$, and on $\mathbf{n}_{e}$, which is the normal to $e$ oriented outward from $T$ and inward into $T_{e}$. They also depend on $\widetilde{\mathbf{U}}_{T}$ and $\widetilde{\mathbf{U}}_{T_{e}}$, which are the piecewise polynomial representations of the solution in $T$ and $T_{e}$. This functional dependence implies the usage of pointwise values of the approximate solution at quadrature nodes on $e$. These values are reconstructed from the cell averages by an interpolation procedure at each time step and a multidimensional slope limiter must be considered to take under control the numerical oscillations, see the appendix of Reference [12].

The integral advective term $\mathbf{G}_{e}$ is discretized by a standard upwind flux splitting approach, while the integral dispersion term $\mathbf{H}_{e}$, which involves second derivatives in space, by a central differentiation algorithm. Further details about the derivation and the accuracy of this method are discussed in Reference [15]. 
The numerical flux function $\mathbf{F}_{e}^{(b c)}$ at the boundary edge $e=\partial T \cap \partial \Omega$ depends on the trace $\left.\widetilde{\mathbf{U}}_{T}\right|_{e}$ of the reconstructed solution $\widetilde{\mathbf{U}}_{T}$ within the unique boundary triangle $T$, and in some suitable form on a set of external data $\mathbf{U}_{e}^{(b c)}$.

The integral source term $\mathbf{S}_{T}\left(\widetilde{\mathbf{U}}_{T}\left(\mathbf{x}_{T, q}\right)\right)$ is approximated by a surface quadrature rule with nodes $\left\{\mathbf{x}_{T, q}\right\}$ within the triangle $T$ and weights $\left\{\omega_{T, q}\right\}$.

The time-marching scheme is obtained by approximating the time derivative of $\mathbf{U}_{T}$ - which appears in the first term in the semi-discrete formulation (8) - by first-order finite differences

$$
\left.\frac{d \mathbf{U}_{T}(t)}{d t}\right|_{t=t^{n}} \approx \frac{\mathbf{U}_{T}^{n+1}-\mathbf{U}_{T}^{n}}{\Delta t}
$$

where $\mathbf{U}_{T}^{n+1}$ and $\mathbf{U}_{T}^{n}$ are the cell-averaged solutions in $T$ at times $t^{n+1}$ and $t^{n}$, and $\Delta t=$ $t^{n+1}-t^{n}$. This yields a full discrete semi-implicit scheme where resulting symmetric linear algebraic problem is solved by a standard Krylov solver, such as a preconditioned conjugate gradient method. Higher (second) order accuracy in time is attainable by a semi-implicit Runge-Kutta method, built by two distinct stages of the same form [12].

\subsection{The Mixed-Hybrid Discretization of the Darcy's Equation}

The coupled system of equations (2) in the unknowns $p$ and $\mathbf{u}$ is discretized by a mixed-hybrid finite element approach. For a detailed exposition of mixed and mixed-hybrid finite element methods we refer the reader to References [5,6], while for the description of the numerical formulation adopted in this work we refer to Reference [12].

In the mixed-hybrid finite element method adopted in the present work we approximate the velocity field by using the lowest-order $\mathcal{R T}_{0}$ discontinuous elements, which is composed by two-dimensional functions whose restriction to any mesh triangle $T$ is of the form

$$
\left.\mathbf{u}\right|_{T} \approx \alpha_{T}\left(\begin{array}{c}
x \\
y
\end{array}\right)+\left(\begin{array}{c}
\beta_{T} \\
\gamma_{T}
\end{array}\right)
$$

where the real scalar coefficients $\alpha_{T}, \beta_{T}$ and $\gamma_{T}$ depend on the triangle $T$. The pressure field is approximated by the triangle-based piecewise constant functions while the pressure trace over each cell-interface by the edge-based piecewise constant ones.

With respect to Reference [12], the present work differs substantially in the choice of the discrete functional space used for the approximation of the velocity field $\mathbf{u}$. We use here the lowest-order $\mathcal{R} \mathcal{T}_{0}$ discontinuous elements instead of the $\mathcal{B D} \mathcal{M}_{1}$ ones of Reference [12], where a full linear dependence on the position is considered. Notice also that the continuity condition of the normal component of the velocity flux is relaxed, and a weaker condition is imposed by a set of suitable Lagrange multipliers which approximates the pressure traces.

We experienced in fact that $\mathcal{R T}_{0}$ elements offers a satisfactory accuracy level at a reduced computational cost with respect to $\mathcal{B D} \mathcal{M}_{1}$ elements, see Reference [13]. These latter ones are formally more accurate but also significantly more demanding from a computational viewpoint because they involve twice the number of unknowns to be stored and calculated.

This issue is particularly important because in this work the pressure and the velocity fields are iteratively updated at each time step, see Reference [11], while in the work described in Reference [12] they were calculated only once at the beginning of each simulation and then used to transport passively the concentration fields of the contaminant species. 


\subsection{Reaction Source Terms and Microbial Population Equation}

The reaction terms described in equations (6) are computed by solving a set of nodewise nonlinear systems via a Newton iterative method with fractional multistep integration scheme, see Reference [12].

\section{NUMERICAL EXPERIMENTS}

In this section we illustrate the performance of the proposed mathematical and numerical model in predicting the effectiveness of a human remediation intervention to reduce the contaminant concentration of a polluted aquifer. The aquifer is characterized by a constant porosity $\phi=0.3$ and a heterogeneous isotropic transmissivity, whose principal values are assumed to be constant on each triangle of the computational mesh, and differ triangle by triangle in the range between $10^{-5}$ and $1 \mathrm{~m}^{2} /$ day, in accord with an equiprobability stochastic distribution.

The first test case that we present in this paper consists in the initial soil contamination phase and is labeled by $T 1$. The soil contamination is due to the leakage of Cyclo-AromaticHydrocarbons, CAH, which forms a plume transported by the groundwater flow field and spread in the saturated aquifer.

The next three test cases, labeled by $T 2, T 3$ and $T 4$, describes three possible interventions for the remediation phase. Basically, we consider a network of pumping wells that extract the polluted water and convey it to a treatment plant, where the contaminant is removed. The purified water may be enriched in oxygen and nutrients to stimulate soil bacterial growth and is then re-injected in the aquifer via a network of injection wells. Figure 1 sketches the water treatment procedure.

All of the wells can be selectively used either in injection or in extraction mode and are all supposed to be connected via pipelines to the water treatment plant. Point "A" is also the location of the leaking tank when contamination occurs. The proper position and configuration of the wells has been chosen by investigating their capability of intercepting the contaminated plume transported by the groundwater flow in a set of preliminary simulations.

These simulations are based on the four species model proposed by Molz et al. [21] and Widdowson et al. [27], and described in [12]. For the sake of completeness, we report the model in the final appendix, giving also the values of the parameters used in the simulations. The contaminant CAH is the organic substrate $S$ of the model, while the other species involved are the dissolved oxygen in the soil, $O$, some compounds chemically based on nitrates, $N$, and some ammonia-based compounds which constitute a generic nutrient supply $A$. The initial concentrations of these species follow a random distribution, with values in the ranges reported in Table I.

Figure 2 depicts the benchmark case and the position of the wells on the aquifer - labels "A" through "M" - in the remediation phase. A constant gradient of $\Delta p / \Delta x=0.04$ is superimposed on the subsurface bulk flow field in the aquifer, which is thus oriented along the $x$ direction. These model problems are completely defined by a suitable Dirichlet boundary 
Table I. Microcolony concentration ranges (in $\mathrm{g} / \mathrm{m}^{3}$ ) at the beginning of the simulation $(t=0)$.

\begin{tabular}{|c|c|c|}
\hline$c_{S}(\mathrm{t}=0)$ & $c_{O}(\mathrm{t}=0)$ & $c_{N}=c_{A}(\mathrm{t}=0)$ \\
$\left(\mathrm{g} / \mathrm{m}^{3}\right)$ & $\left(\mathrm{g} / \mathrm{m}^{3}\right)$ & $\left(\mathrm{g} / \mathrm{m}^{3}\right)$ \\
{$[0.1,1]$} & {$[0.1,1]$} & 1000 \\
\hline
\end{tabular}

Table II. Simulation run parameters

\begin{tabular}{||r||r|r|r|r||}
\hline \multirow{2}{*||}{ Parameter } & \multicolumn{4}{|c||}{ Run Label } \\
\cline { 2 - 5 } & $\mathrm{T} 1$ & $\mathrm{~T} 2$ & $\mathrm{~T} 3$ & $\mathrm{~T} 4$ \\
\hline$\Delta t($ days $)$ & 0.5 & 0.2 & 0.05 & 0.05 \\
$T_{\max }($ days $)$ & 410 & 2800 & 500 & 700 \\
\hline
\end{tabular}

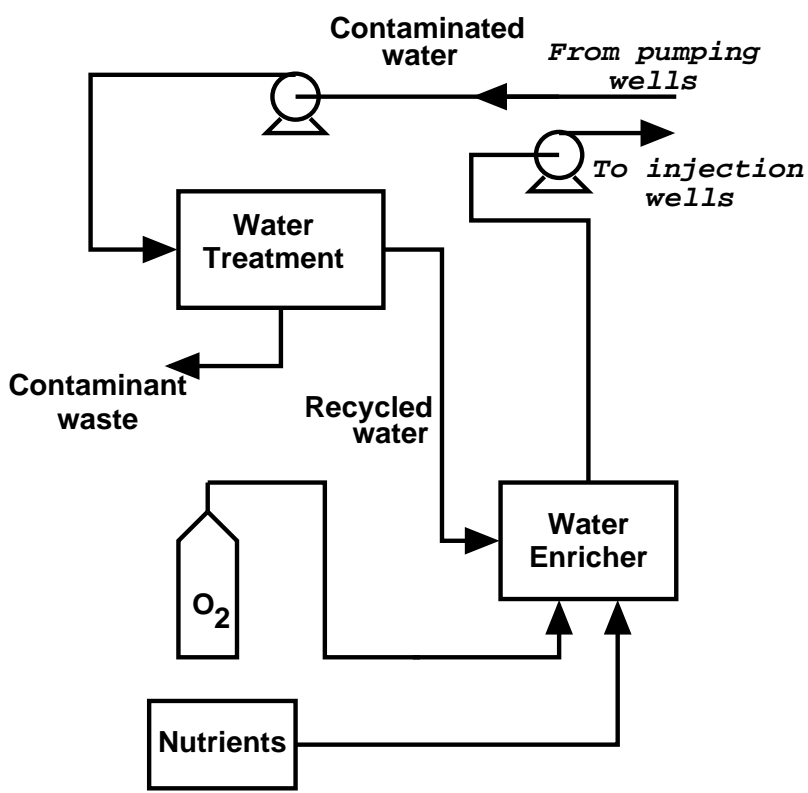

Figure 1. Technical scheme of the remediation plant installed aside of the well network for polluted water treatment and clean water enrichment.

condition, with an hydraulic pressure head given as a function of $x$. For each simulation run, we report in Table II the time step $\Delta t$ and the final time $T_{\max }$ at which the simulation ends up.

During the initial pollution phase $(T 1)$, the plume of $\mathrm{CAH}$ spreads with an irregular, or "fingered", front because of the stochastic soil heterogeneity which establishes several preferential paths. Figure 3 illustrates the situation at the intermediate time $t=410$ days, when the contaminant reaches the boundary of the aquifer and begins to dissolve into the water 


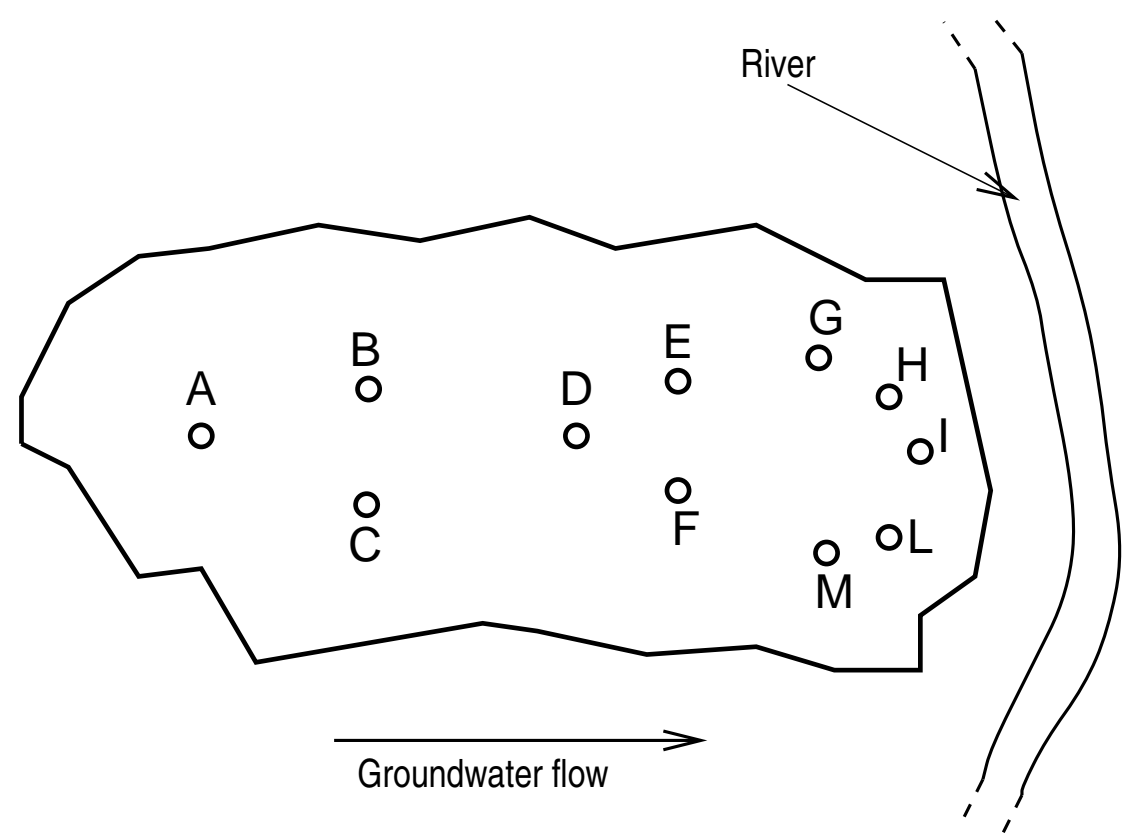

Figure 2. Planar sketch of the well locations.

of the confining river. A steady state solution is reached at $t=600$ days, shown in figure 3 , when the contaminant plume does not spread further and the transport of the contaminant along some preferential paths is thus established.

Three different possible intervention strategies have been investigated. Remediation starts after $t=410$ days since the pollution started, that is before the contaminant reaches the river confining with the aquifer. We suppose that the contaminant source is removed and the clean-up of the soil is performed by using a network of extraction/injection wells. For all of the simulations we will show the spatial distribution of the organic substrate, CAH, and of the dissolved oxygen, DO - the species $\mathrm{O}$ in our four species model.

In the simulation $T 2$ we consider the so-called pump-and-treat method. The wells $\mathrm{G}$ through $\mathrm{M}$ of the pipeline network are active and extract the contaminated water, which is then conveyed to water treatment plants.

The flow boundary conditions are the same as for the soil contamination phase, except at the location corresponding to the pumping wells: here, a decrease of $0.5 \mathrm{~m}$ in pressure head with respect to the natural gradient condition is imposed in order to modify the flow pattern for contaminant recovery. As in the soil contamination phase, just one chemical species is considered in the simulation, by neglecting the effects of the other species.

The result of the simulation is shown in figure 5 at the intermediate time $t=1490$ days, that is about 4 years after the removal of the contaminant source. Heterogeneity still affects the effectiveness of the removal, but its spreading effect is not as evident as in the first phase. 
The computer simulation is terminated at $t=2500$ days - about 7 years. It is worth noticing that the removal of the organic contaminant plume has not yet been fully completed.

The bioremediation interventions considered in this paper - simulations $T 3$ and $T 4$ - are essentially based on the stimulation of the growth of the subsurface bacterial population by increasing the concentration of oxygen and nutrients dissolved in soil. We assume that these chemical substances be directly supplied via injection of "enriched water" into the aquifer. Part of the wells are, thus, used for extracting the contaminated water to be conveyed to treatment plants. Part or all of the remaining wells are used for re-injecting water with chemical additives into the soil. The effectiveness of this strategy strongly depends on the extractioninjection operational mode chosen in the well configuration pattern, which is different for the two simulations $T 3$ and $T 4$.

In the configuration of the simulation $T 3$ the wells $\mathrm{A}$ through $\mathrm{D}$ work in injection mode an overpressure of $0.5 \mathrm{~m}$ is imposed. Nutrients and nitrates are delivered in excess, since their solubility in water is much larger than the one of oxygen. This latter chemical is kept at a constant concentration of $20 \mathrm{mg} / \mathrm{l}$. The remaining wells work in extraction mode, at the same pressure condition of the "pump-and-treat" method.

The result of this simulation is shown at the intermediate time $t=178$ days after the removal of the contaminant started. No significant improvements in contaminants removal are achieved from this configuration of pumping and injecting wells after $t=360$ days. A "deadzone" develops in the triangular region defined by the wells "F-G-H", all working in pumping conditions. It is evident by comparing figures 8 and 6 the difference in the contaminant removal between the current simulation and the preceding one, particularly where dissolved oxygen has been delivered.

In the configuration of the simulation $T 4$ wells $\mathrm{G}$ through $\mathrm{M}$ still work in extraction mode, wells $\mathrm{E}$ and $\mathrm{F}$ inject nutrients and oxygen, while wells $\mathrm{A}$ through $\mathrm{D}$ are dismissed. figure 9 shows the contaminant and dissolved oxygen distributions at the intermediate time $t=600$ days. We remark that the major part of the residual contaminant mass in the aquifer is removed. A complete removal of the contaminant is achieved at the final time $t=875$ days, as illustrated by Figure 9 .

Finally, figure 11 reports the residual contaminant mass in the aquifer as a decreasing function of time and summarizes the performance of the different remediation approaches $T 2$, $T 3$ and T4. This figure emphasizes how the bioremediation strategy can be more effective than the simple pump-and-treat method. Although both intervention strategies $T 2$ and $T 4$ achieves an almost complete removal of the contaminant, the remediation time is very different in the two cases. Nevertheless, bioremediation can also be sensitive to the well configuration network chosen for the intervention, as shown by the performance curve $T 3$.

\subsection{Model Performances}

In this section we report some information about the costs in term of CPU time of the computer simulations.

All the simulations described in the previous section were run on a computational mesh composed of about 5000 triangles using an IBM RISC 6000/390 machine. The simulations involving the full four-species model are very expensive and take about 12 hours, while singlespecies calculations require typically 80-90 minutes. 
Table 2: CPU Costs (minutes)

\begin{tabular}{||r||r|r|r|r||}
\hline \multirow{2}{*}{ Parameter } & \multicolumn{4}{|c}{ Run Label } \\
\cline { 2 - 5 } & T1 & T2 & T3 & T4 \\
\hline$C P U$ & 80 & 90 & 720 & 720 \\
\hline
\end{tabular}

The CPU cost is quite high in the former case because all of the non-linear interactions among the different species must be taken into account. A $4 \times 4$ non-linear systems must be solved for each cell at each time step, by using a Newton iterative scheme, which takes approximately $40 \%$ of the total CPU costs. Moreover, the finite volume method requires a limited piecewise-linear reconstruction of each unknown field to ensure $2^{\text {nd }}$-order accuracy in space. The limiting procedure is needed to ensure monotonicity of reconstructed gradients and to preserve non-linear stability, see Reference [12] for details. The computational cost of the reconstruction procedure is also significant, being about $35 \%$ of the total CPU costs of the simulation. This increment is evident when the full species model is considered instead of the single-species one.

CPU costs are also influenced by the way the simulation is run. For instance, in the initial pollutant phase $T 1$, the contaminant is transported by a steady groundwater flow field, which is computed only once at the beginning of the run. Instead, the simulation $T 2$ stills involves a single-species model, but makes usage of a transient groundwater velocity field, which is updated every 50 transport steps, thus resulting in a more expensive computation.

\section{CONCLUSIONS}

In this work we illustrated a numerical model to investigate the bioremediation process in heterogeneous saturated aquifers and its applications in devising different intervention strategies on a field-size scenarios. The method is particularly suitable in detecting dead-zones due to the heterogeneity of the medium and dependent on the well location and operational mode.

Our approach is based on the discretization of a multispecies transport model coupled with a bacterial degradation kinetics of Monod type. The microcolony description of bacterial activity is considered. The bulk flow velocity is approximated by a mixed-hybrid finite element method while the species transport equations are discretized by using a semi-implicit cell-center finite volume scheme.

The performance of the method are assessed by studying the contamination process and several remediation strategies on a realistic subsurface scenario.

A comparison of the numerical experiments reported in this work clearly illustrates the advantage of a combined biological-hydraulic intervention with respect to the simple hydraulic one in the case of a stochastically heterogeneous soil. The remediation time in the former case is shown to be about half the one of the latter case. This fact implies that the operational costs may be substantially reduced.

When the aquifer is strongly heterogeneous, preferential flow zones may appear and large quantities of contaminant may remain isolated if the simplest pump-and-treat remediation strategy is adopted. Contaminant trapping effects due to heterogeneities may also be 
present when remediation is enhanced by bacterial activity. In such a case, an optimal well configuration has a dramatic impact on the effectiveness of the human intervention. For these reasons, it is evident that a better understanding of how and where trapping zones appear is critical in devising an effective remediation strategy.

In order to study the near-source contamination zone, that is the the zone surrounding an organic contaminant spill, a multiphase model is needed, because an organic phase appears. It is informative to say that some preliminary work $[14,11]$ has been performed by the authors to develop a suitable numerical approach to multiphase simulations as well as considering the problem of pore-clogging in biofilm models. However, these topics will be the issue of future work.

\section{ACKNOWLEDGEMENT}

The work of Claudio Gallo has been financially supported by Sardinian Regional Authorities. The authors would like to thank Dr. Fabio Bettio for his help in visualization and Dr. Enrico Bertolazzi (University of Trento, Italy) for his careful reading of the preliminary version of the paper and his useful suggestions. The unstructured Delaunay grids were generated by the mesh generator TRIANGLE, a code implemented by Shewchuck, see the URL:http://almond.srv.cs.cmu.edu/afs/cs/project/quake/public/www/triangle.html.

\section{REFERENCES}

1. J. E. Bailey and D. F. Ollis. Biochemical Engineering Fundamentals. McGraw-Hill, New York, 1977.

2. P. Baveye and A. Valocchi. An evaluation of mathematical models of the transport biologically reacting solutes in saturated soils and aquifers. Water Resour. Res., 25(6):1413-1421, 1989.

3. J. Bear. Hydraulics of Groundwater. McGraw-Hill, New York, 1979.

4. L. Bergamaschi, C. Gallo, G. Manzini, C. Paniconi, and M. Putti. A mixed finite-elements/TVD finitevolumes scheme for saturated flow and transport in groundwater. In Cecchi et al., editor, Finite Elements in Fluids, pages 1223-1232, Padova, Italy, 1995.

5. F. Brezzi and M. Fortin. Mixed and Hybrid Finite Element Methods. Springer Verlag, Berlin, 1991.

6. G. Chavent and J. E. Roberts. A Unified Physical Presentation of Mixed, Mixed-Hybrid Finite Elements and Standard Finite Difference Approximation for the Determination of Velocities in Waterflow Problems. Advances in Water Resources, 14(6):329-348, 1991.

7. P. G. Ciarlet. The finite element method for elliptic problems. North-Holland Publishing Company, Amsterdam, Holland, 1980.

8. E. L. Davis. How heat can enhance in-situ and aquifer remediation: important chemical properties and guidance on choosing the appropriate technique. Technical Report EPA/540/S-97/502, US EPA, Technology Innovation Office, Office of Solid Waste and Emergency Response, US EPA, Washington, DC, 1997.

epa96state

9. Environmental Protection Agency. State policies concerning the use of injectants for in situ ground water remediation. Technical Report EPA/542/S-96/001, US EPA, Technology Innovation Office, 1996.

10. R. A. Freeze and J. A. Cherry. Groundwater. Prentice-Hall, New Jersey, 1979.

Freeze:1979 Gallo:2000

Gallo: 1997

Gallo: 1998

allo-Manzini:2000

11. C. Gallo and S. M. Hassanizadeh. Influence of biodegradation on napl flow and dissolution in groundwater. In L. R. Bentley et al., editor, Computational Methods in Water Resources XIII, Volume 1, pages 129-136. A. A. Balkema, Rotterdam, Holland, 2000.

12. C. Gallo and G. Manzini. A mixed finite element/finite volume approach for solving biodegradation transport in groundwater. International Journal of Numerical Methods in Fluids, 26:533-556, 1998.

13. C. Gallo and G. Manzini. 2-d numerical modeling of bioremediation in heterogeneous saturated soils. Transport in Porous Media, 31:67-88, 1998b.

14. C. Gallo and G. Manzini. A fully coupled numerical model for two-phase flow with contaminant transport and biodegradation kinetics. Technical report, IAN-1163, 1999. to appear in Comm. Int. Num. Meth. Engr. 
:technical :report

Hassanizadeh : 1999

Kelly : 1996

Kindred:1989

Lensing: 1994

epa95material

Moltz: 1986

insitubio: 1993

cleanup: 1994

epa92gen

epa92tce

Taylor: 1990

Widdowson : 1988
15. C. Gallo and G. Manzini. Finite volume/mixed finite element analysis of pollutant transport and bioremediation in heterogeneous saturated aquifers. Technical report, IAN-Tech. Rep., 2001.

16. S. M. Hassanizadeh. Upscaling equations of solute transport and biodegradation in soils. Technical report, Dept. of Civil Engineering and Geosciences, TUDelft, The Netherlands, 1999.

17. W. R. Kelly, G. M. Hornberger, J. S. Herman, and A. L. Mills. Kinetics of BTX biodegradation and mineralization in batch and column systems. J. Contam. Hydrol., 23:113-132, 1996.

18. J.S. Kindred and M.A. Celia. Contaminant transport and biodegradation: 2. conceptual model and test simulations. Water Resources Research, 25(6):1149-1159, 1989.

19. J. J. Lensing, M. Vogt, and B. Herrling. Modeling biologically mediated redox processes in the subsurface. J. Hydrol., 159:125-143, 1994

20. D. R. McCaulou, D. G. Jewett, and S. G. Huling. Nonaqueous phase liquids compatibility with materials used in well cnstruction, sampling, and remediation. Technical Report EPA/540/S-95/503, US EPA, Technology Innovation Office, Office of Solid Waste and Emergency Response, US EPA, Washington, DC, 1995.

21. F. J. Molz, M. A. Widdowson, and L. D. Benefield. Simulation of microbial growth dynamics coupled to nutrient and oxygen transport in porous media. Water Resources Research, 22(8):1207-1216, 1986.

22. National Research Council. In Situ Bioremediation: When Does It Work? National Academy Press, Washington, D.C., 1993.

23. National Research Council. Alternatives for Ground Water Cleanup. National Academy Press, Washington, D.C., 1994.

24. R. R. Ross. General methods for remedial operations performance evaluations. Technical Report EPA/600/R-92/002, US EPA, Technology Innovation Office, Office of Solid Waste and Emergency Response, US EPA, Washington, DC, 1992.

25. H. H. Russel, J. E. Matthews, and G. W. Sewell. TCE removal from contaminated soil and groundwater. Technical Report EPA/540/S-92/002, US EPA, Technology Innovation Office, Office of Solid Waste and Emergency Response, US EPA, Washington, DC, 1992.

26. S. W. Taylor and P. R. Jaffè. Substrate and biomass transport in a porous medium. Water Resour. Res., 26(9):2181-2194, 1990.

27. M. A. Widdowson, F. J. Molz, and L. D. Benefield. A numerical transport model for oxygen- and nitratebased respiration linked to substrate and nutrient availability in porous media. Water Resources Research, 24(9):1553-1565, 1988. 


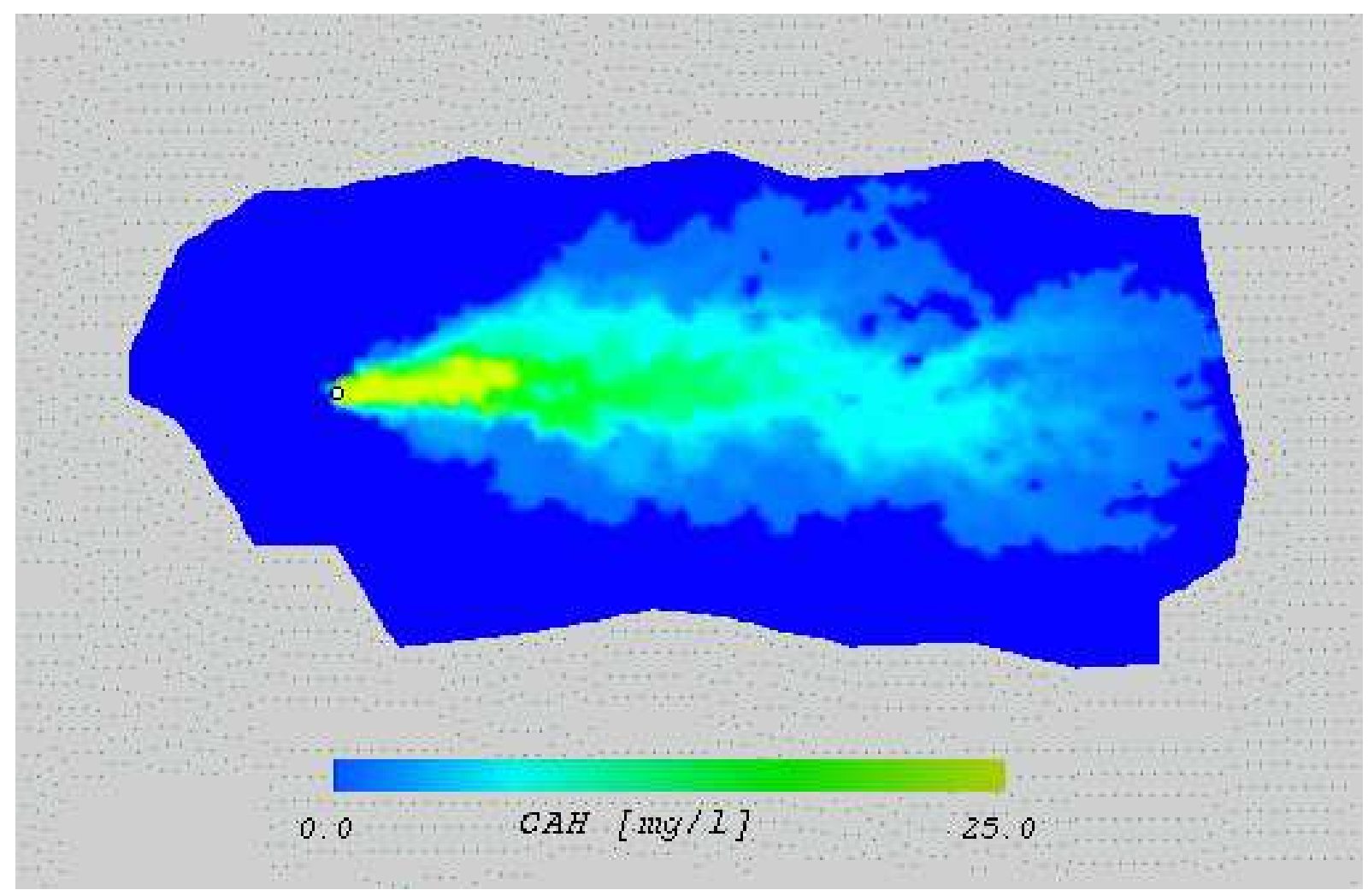

Figure 3. Contamination phase (T1): pollutant concentration at $t=410$ days 


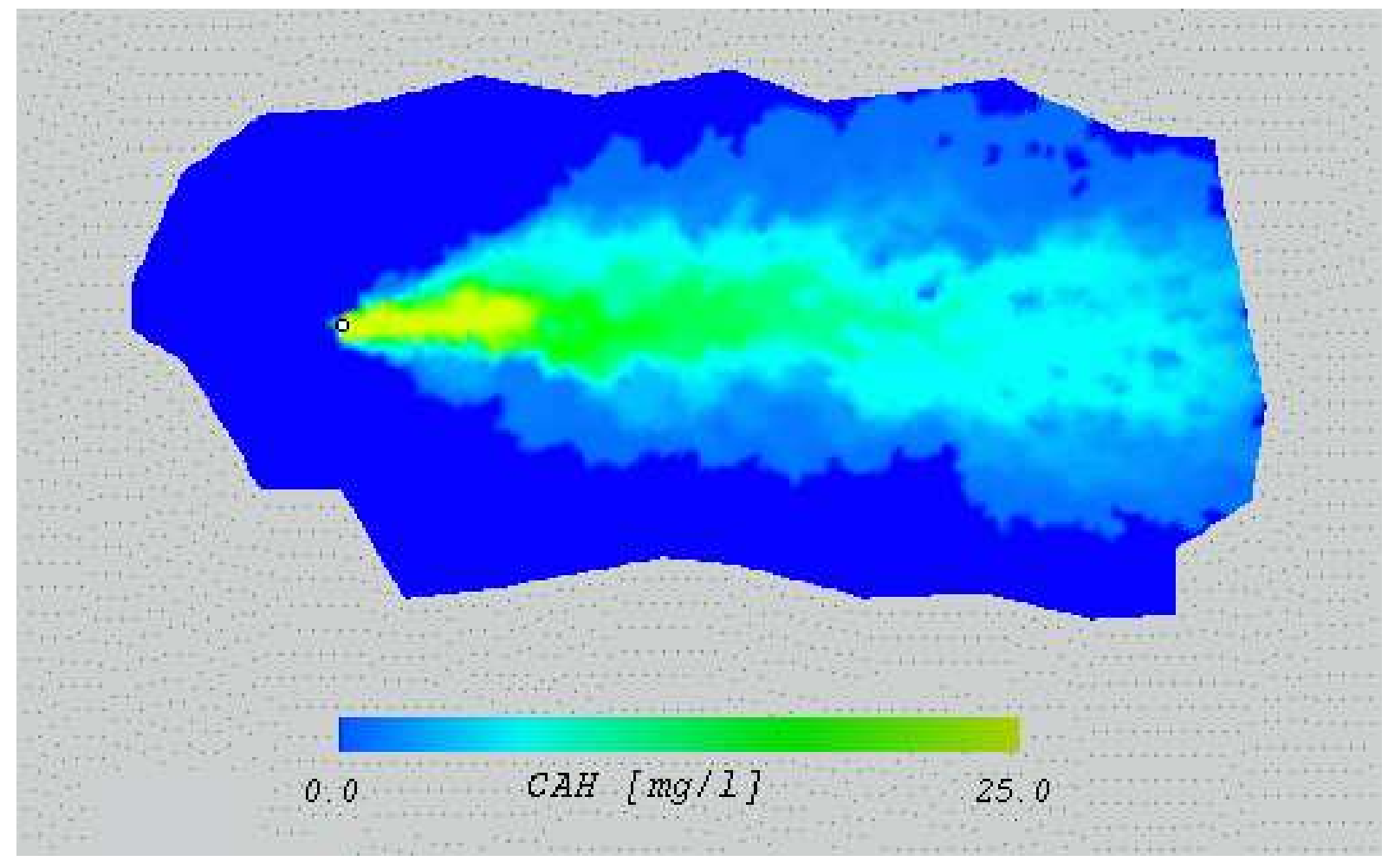

Figure 4. Contamination phase (T1): pollutant concentration at $t=600$ days - steady state 


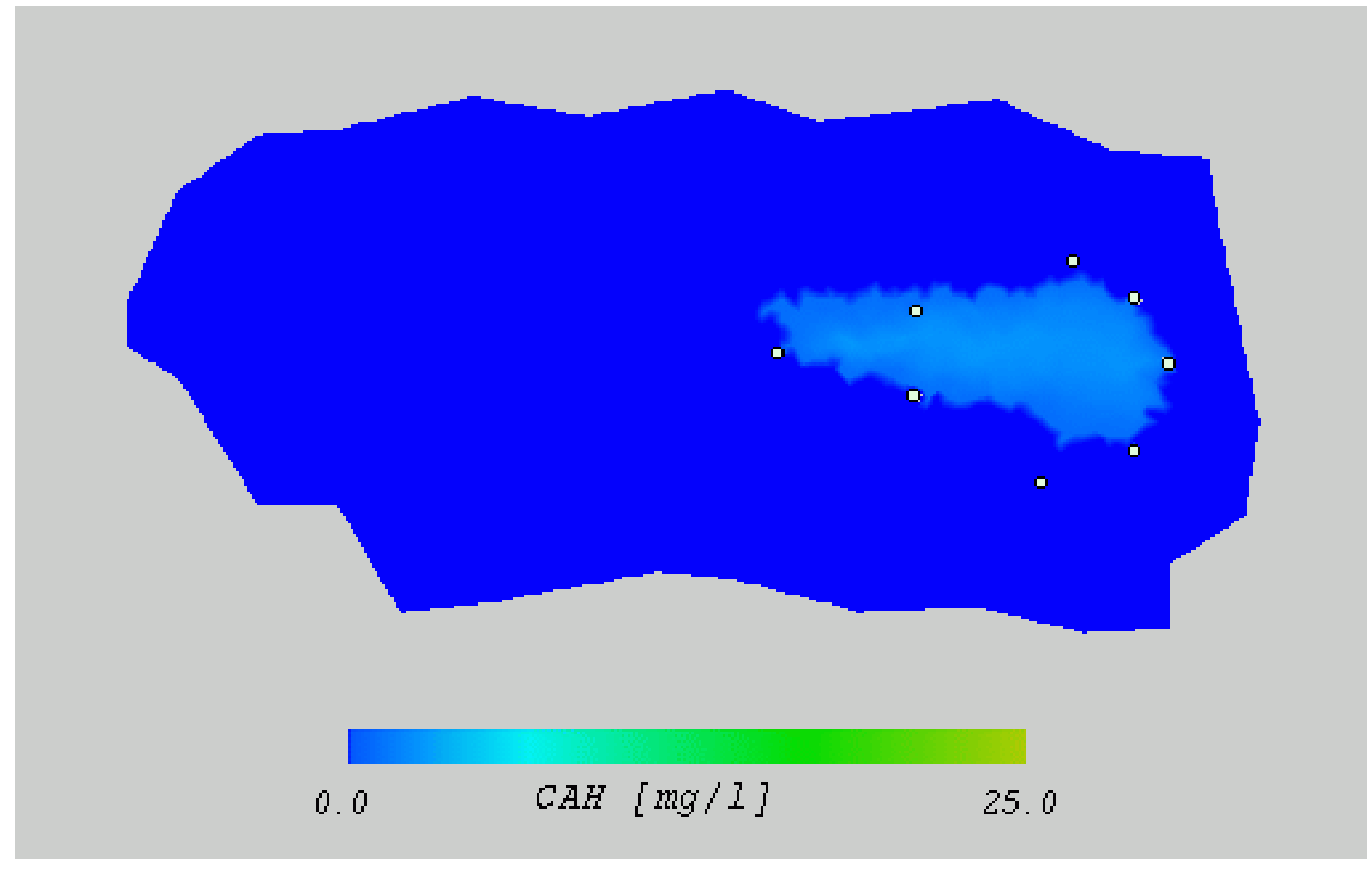

Figure 5. Pump-and-treat remediation (T2): concentration at $t=1450$ days 


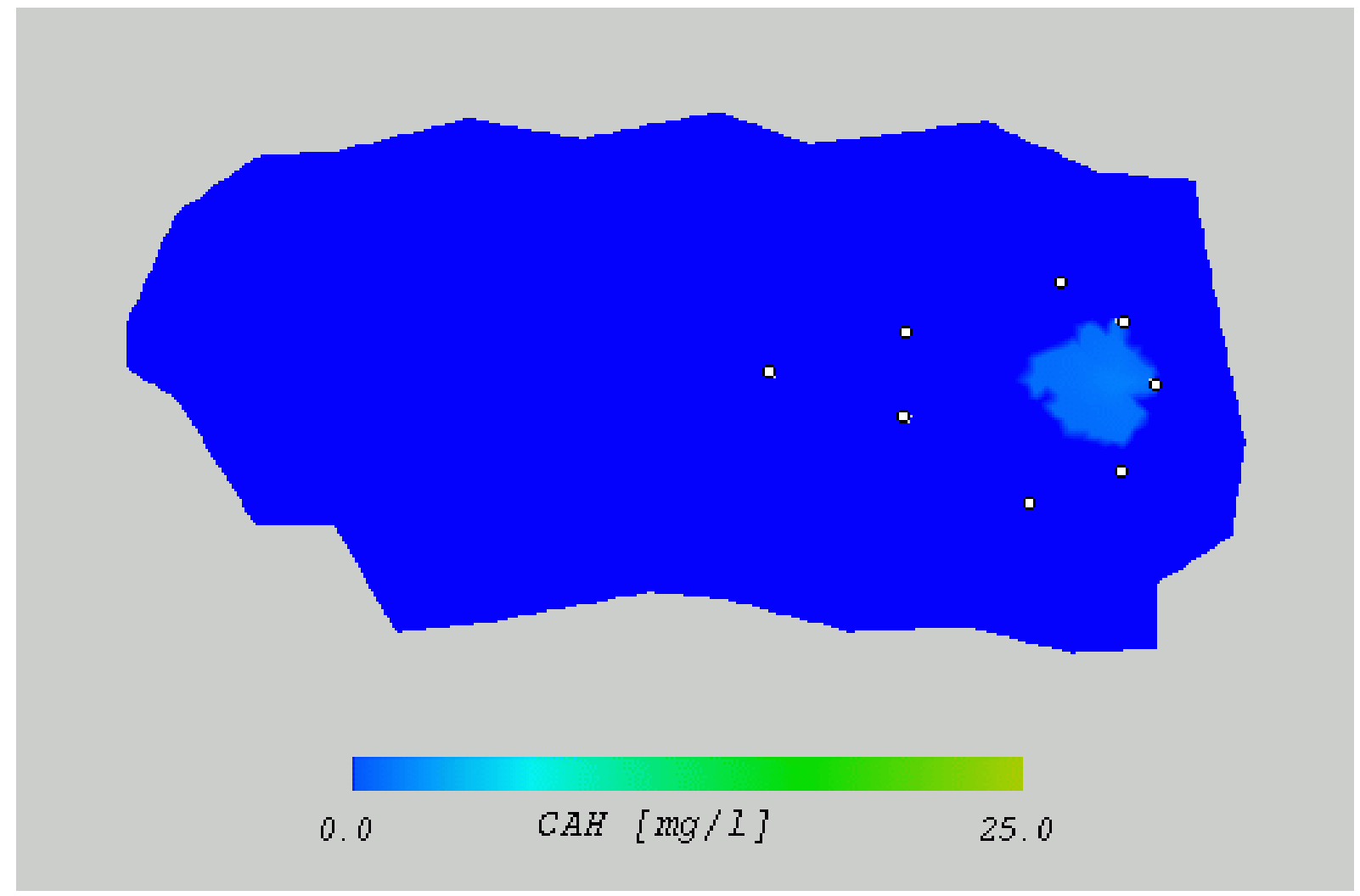

Figure 6. Pump-and-treat remediation (T2): concentration at $t=2500$ days 


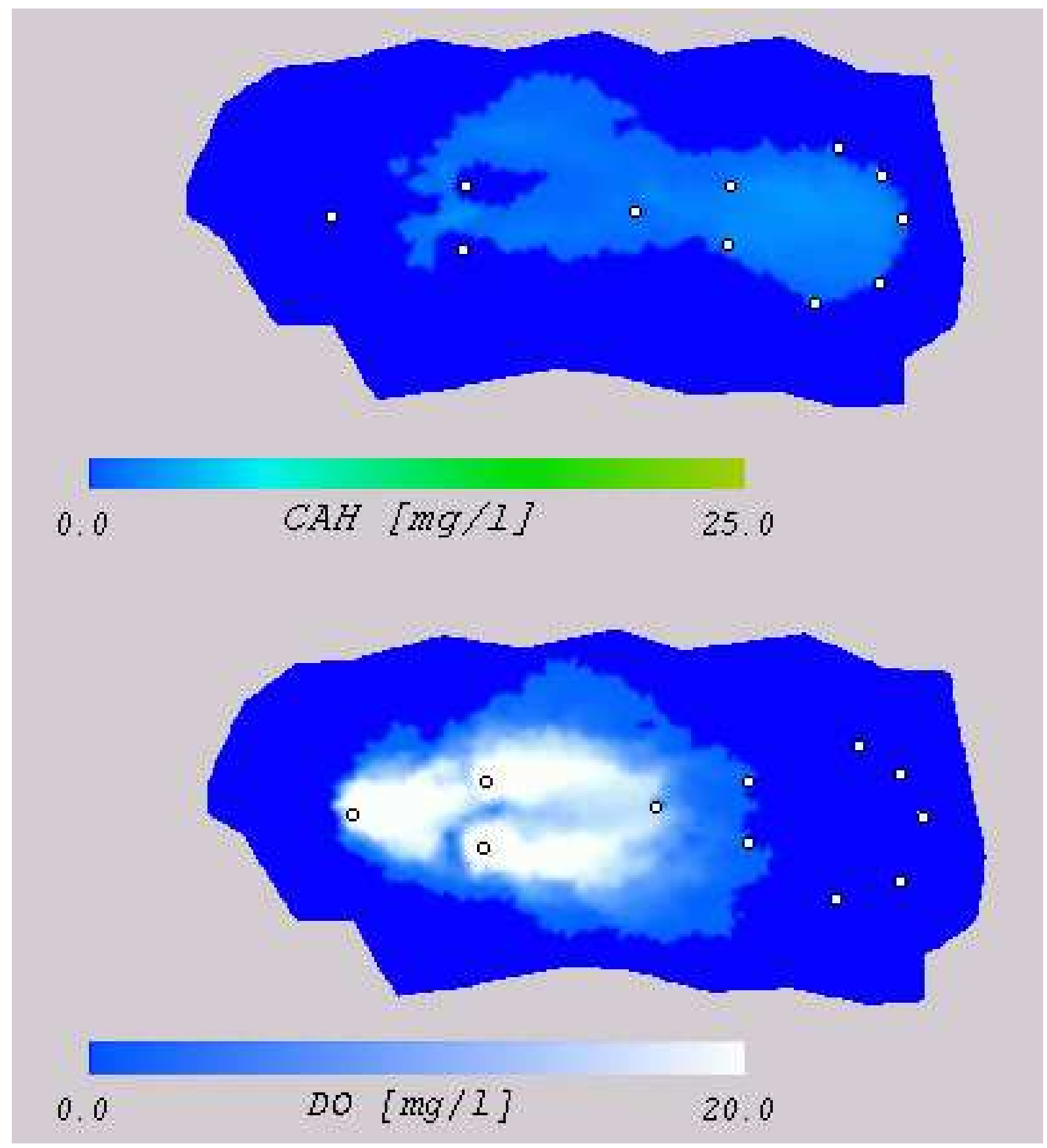

Figure 7 . Bioremediation by $1^{\text {st }}$ well configuration (T3): concentration at $t=178$ days 


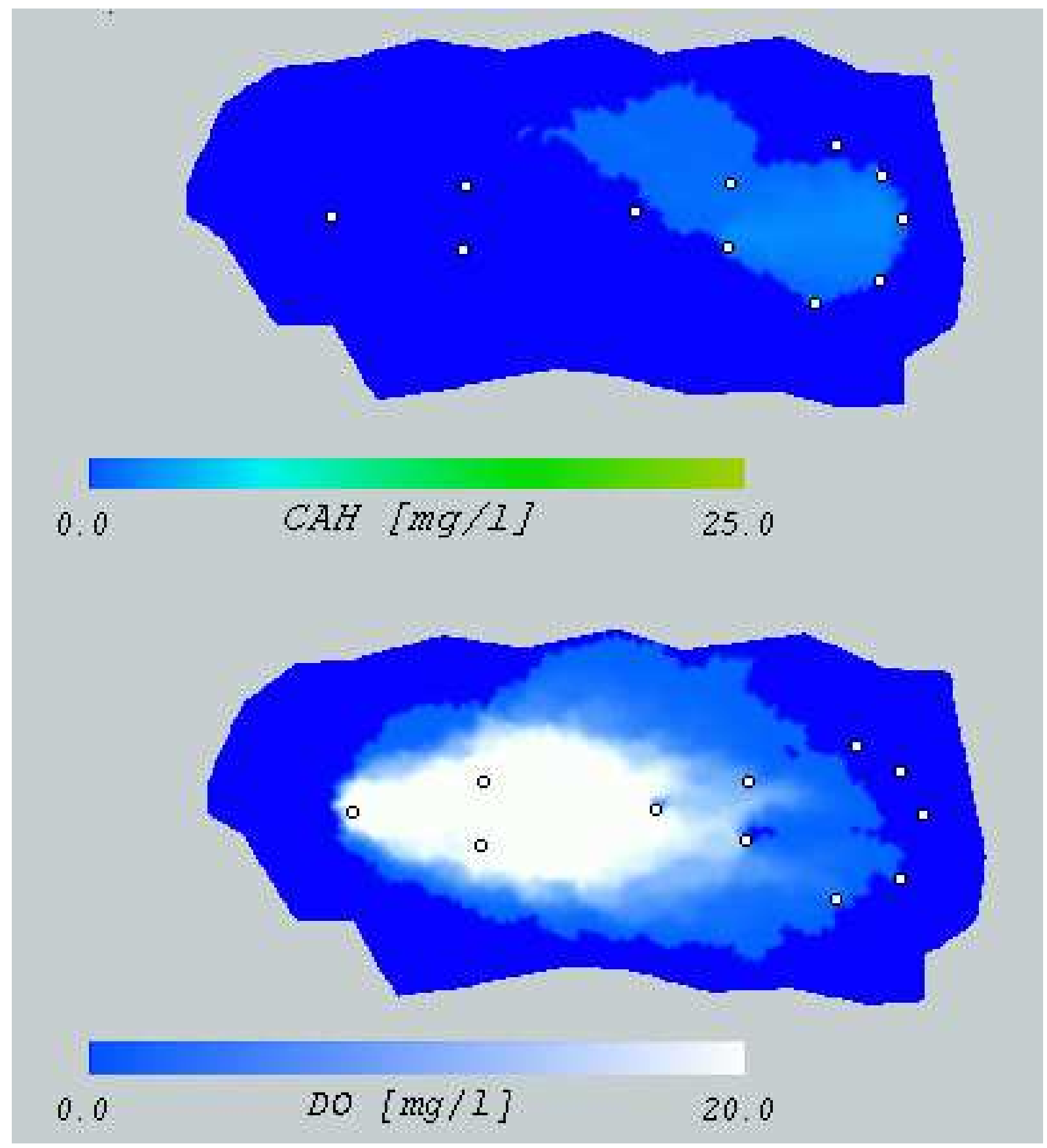

Figure 8 . Bioremediation by $1^{\text {st }}$ well configuration (T3): concentration at $t=360$ days 


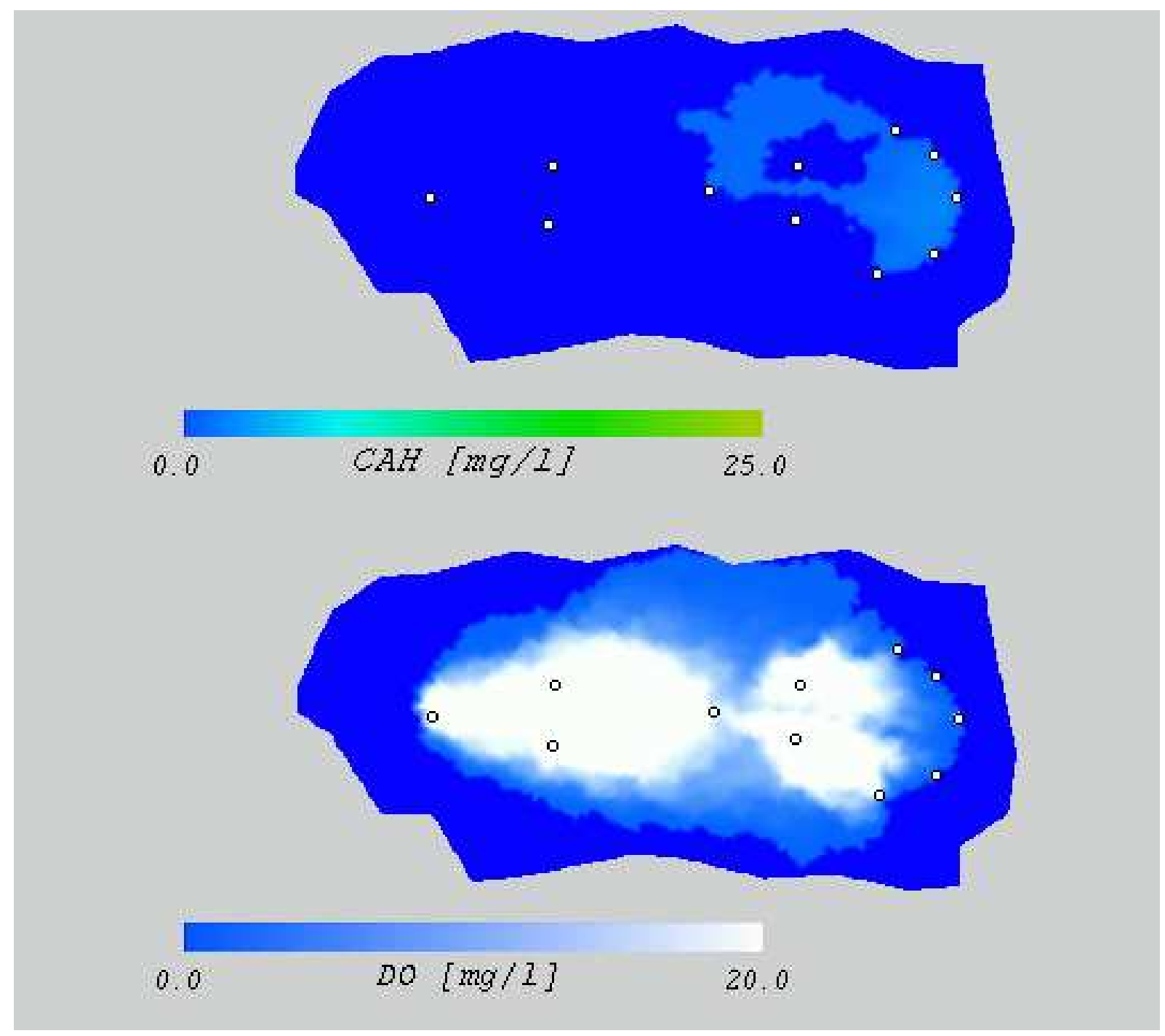

Figure 9 . Bioremediation by $2^{\text {nd }}$ well configuration (T4): concentration at $t=600$ days $(200$ days after the switch) 


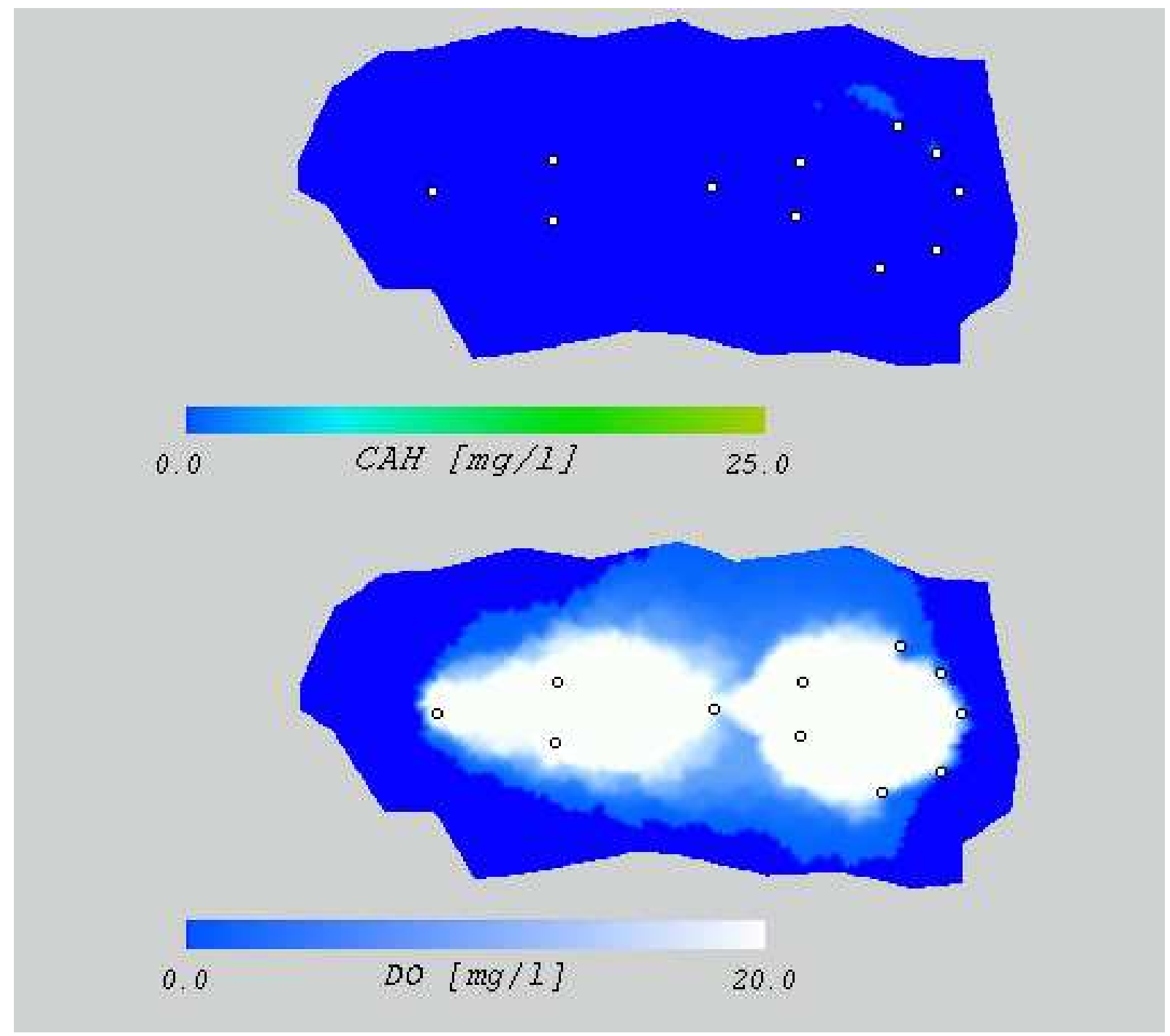
Figure 10. Bioremediation by $2^{\text {nd }}$ well configuration (T4): concentration at $t=875$ days (475 days bio2b
after the switch) 


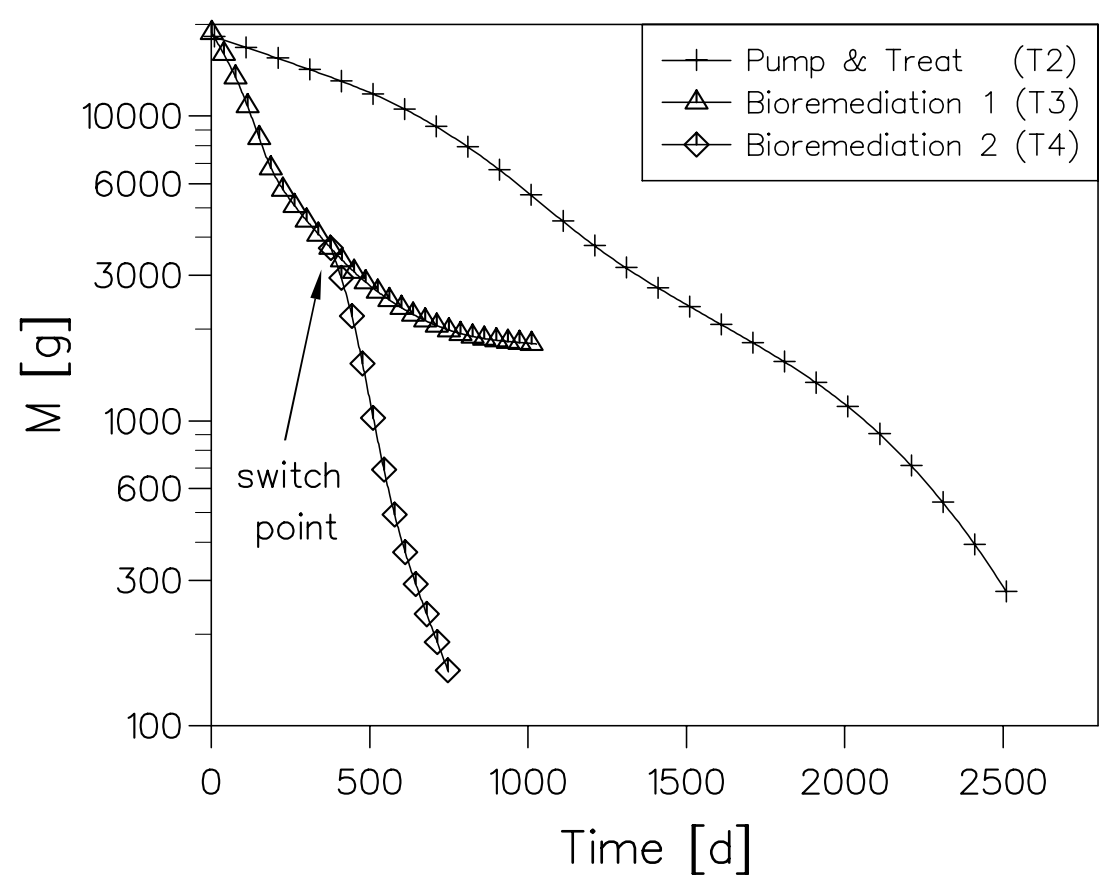

Figure 11. Contaminant residual mass removal vs remediation time. 


\section{APPENDIX}

The kinetic degradation rates of concentrations within microcolonies in (3) for the four species model used in all of the simulations are

$$
\begin{aligned}
\kappa_{S} A_{c} \frac{\left(C_{S}-c_{S}\right)}{\delta} & =m_{c} Y_{S, O} \mu_{0, O}\left[\frac{c_{S}}{K_{S, O}+c_{S}}\right]\left[\frac{c_{O}}{K_{O}+c_{O}}\right]\left[\frac{c_{A}}{K_{A, O}+c_{A}}\right] \\
& +m_{c} Y_{S, N} \mu_{0, N}\left[\frac{c_{S}}{K_{S, N}+c_{S}}\right]\left[\frac{c_{N}}{K_{N}+c_{N}}\right]\left[\frac{c_{A}}{K_{A, N}+c_{A}}\right] I_{b}^{1} \\
\kappa_{O} A_{c} \frac{\left(C_{O}-c_{O}\right)}{\delta} & =m_{c} Y_{O} \mu_{0, O}\left[\frac{c_{S}}{K_{S, O}+c_{S}}\right]\left[\frac{c_{O}}{K_{O}+c_{O}}\right]\left[\frac{c_{A}}{K_{A, O}+c_{A}}\right] \\
& +\alpha_{O} k_{d, O}\left[\frac{c_{O}}{K_{O^{\prime}}+c_{O}}\right] \\
\kappa_{N} A_{c} \frac{\left(C_{N}-c_{N}\right)}{\delta} & =m_{c} Y_{N} \mu_{0, N}\left[\frac{c_{S}}{K_{S, N}+c_{S}}\right]\left[\frac{c_{N}}{K_{N}+c_{N}}\right]\left[\frac{c_{A}}{K_{A, N}+c_{A}}\right] I_{b}^{1}, \\
\kappa_{A} A_{c} \frac{\left(C_{A}-c_{A}\right)}{\delta} & =m_{N} k_{d, N}\left[\frac{c_{N}}{K_{N^{\prime}}+c_{N}}\right] I_{b}^{1}, \\
& +m_{c} Y_{A, N} \mu_{0, N}\left[\frac{c_{S}}{K_{S, O}+c_{S}}\right]\left[\frac{c_{O}}{K_{O}+c_{O}}\right]\left[\frac{c_{A}}{K_{A, O}+c_{A}}\right],\left[\frac{c_{N}}{K_{N}+c_{N}}\right]\left[\frac{c_{A}}{K_{A, N}+c_{A}}\right] I_{b}^{1},
\end{aligned}
$$

and the microbial growth/decay equation is

$$
\begin{aligned}
\frac{1}{N_{c}} \frac{\partial \phi N_{c}}{\partial t} & =\left(\mu_{0, O}\left[\frac{c_{S}}{K_{S, O}+c_{S}}\right]\left[\frac{c_{O}}{K_{O}+c_{O}}\right]\left[\frac{c_{A}}{K_{A, O}+c_{A}}\right]-k_{d, O}\right) \\
& +\left(\mu_{0, N}\left[\frac{c_{S}}{K_{S, N}+c_{S}}\right]\left[\frac{c_{N}}{K_{N}+c_{N}}\right]\left[\frac{c_{A}}{K_{A, N}+c_{A}}\right]-k_{d, N}\right) I_{b}^{1}
\end{aligned}
$$

where

- $\kappa_{S}=1.0310^{-5}\left[\mathrm{~m}^{2} /\right.$ day $], \kappa_{O}=2.1910^{-5}\left[\mathrm{~m}^{2} /\right.$ day $], \kappa_{N}=1.5010^{-5}\left[\mathrm{~m}^{2} /\right.$ day $], \kappa_{A}=1.8610^{-5}$ $\left[\mathrm{m}^{2} /\right.$ day] are the mass exchange coefficients for the bulk flow and the microcolony species concentrations;

- $A_{c}=3.76810^{-10}\left[\mathrm{~m}^{2}\right]$, is the contact area of the microcolony for the mass diffusion process;

- $\delta=5.010^{-4}[\mathrm{~m}]$ is the thickness of the boundary layer between bulk flow and microcolonies;

- $m_{c}=2.8610^{-11}$ is the microcolony mass;

- $\mu_{0, O}=4.34[1 /$ day $]$ and $\mu_{0, N}=2.9$ [1/day] are the specific aerobic and anaerobic growth rates;

- $Y_{S, O}=0.278$ and $Y_{S, N}=0.5$ are the heterotrophic yield coefficients;

- $Y_{O}=0.278[-]$ and $Y_{N}=0.5[-]$ are the coefficients for the oxygen and nitrogen synthesis of heterotrophic biomass;

- $\alpha_{0}=0.0402[-]$ and $\alpha_{N}=0.1[-]$ are the oxygen and nitrogen use-coefficients for maintenance energy of bacteria;

- $Y_{A, O}=0.122$ and $Y_{A, N}=0.122$ are the ammonia-nitrogen coefficients for producing biomass under aerobic and anaerobic conditions;

- $K_{S, O}=40\left[\mathrm{~g} / \mathrm{m}^{3}\right], K_{O}=0.77\left[\mathrm{~g} / \mathrm{m}^{3}\right]$, and $K_{A, O}=1\left[\mathrm{~g} / \mathrm{m}^{3}\right]$ are the substrate, oxygen, and ammonia-nitrogen saturation constants under aerobic conditions;

- $K_{S, N}=40\left[\mathrm{~g} / \mathrm{m}^{3}\right], K_{N}=2.6\left[\mathrm{~g} / \mathrm{m}^{3}\right]$, and $K_{A, N}=1\left[\mathrm{~g} / \mathrm{m}^{3}\right]$ are the substrate, nitrogen, and ammonia-nitrogen saturation constants under anaerobic conditions;

- $K_{O^{\prime}}=0.77\left[\mathrm{~g} / \mathrm{m}^{3}\right]$ and $K_{N^{\prime}}=2.6\left[\mathrm{~g} / \mathrm{m}^{3}\right]$ are the oxygen and nitrogen saturation constants;

- $I_{b}^{0}=1$ and $I_{b}^{1}=K_{b, N} /\left(K_{b, N}+c_{O}\right)$ are the inhibition functions of the oxygen-based and the nitrogen-based respiration, and $K_{b, N}=0.0001\left[\mathrm{~g} / \mathrm{m}^{3}\right]$ is the inhibition coefficient; 
- $k_{d, O}=0.02[1 / \mathrm{day}]$ and $k_{d, N}=0.02[1 / \mathrm{day}]$ are the bacterial death-per-unit-time decay constants for aerobic and anaerobic metabolism. 